DOI: $10.15593 / 2224-9354 / 2020.2 .13$

УДК 620.22.003.13.001.33

\author{
С.М. Вайцехович, Ю.В. Власов
}

\author{
ПОСТРОЕНИЕ МОДЕЛИ ЭКОНОМИЧЕСКОЙ \\ ЭФФЕКТИВНОСТИ ПРОИЗВОДСТВА И ПОТРЕБЛЕНИЯ \\ КОНСТРУКЦИОННЫХ МАТЕРИАЛОВ В МАШИНОСТРОЕНИИ
}

Предложен универсальный экономический критерий, связывающий технологические и эксплуатационные параметры с экономическими показателями, отражающий целесообразность затрат на изготовление и эксплуатацию технической системы во временных параметрах ее жизнеобеспечения. Представлена модель для экономического анализа и оценки применения конструкционных материалов. Критериальный параметр для оценки экономической эффективности технологических процессов у производителя включает в себя коэффициент автоматизации, дисперсию, коэффициент вариации, позволяющий выявить эффективную технологию; у потребителя кратность работы деталей, учет ремонта и замены вышедшей из строя детали. Критериальный параметр позволяет при сравнении альтернативных технологий выявить наиболее рациональную, с точки зрения стабилизации уровня физико-механических свойств материалов на технологических переделах. На основании экспериментальных данных освоения новых конструкционных материалов в энергомашиностроении методом математического моделирования построены таблицы расчета коэффициента соответствия $K_{\sigma}$, учитывающего изменение затрат от уровня технологи-

ческих свойств детали. Разработан универсальный экономический критерий $\frac{K_{t} \cdot K_{N}}{K_{\sigma}}=$ const, ляющий при экономическом анализе технической системы пользоваться только технологическими и временными зависимостями отдельных деталей, входящих в рассматриваемую техническую систему. Разработана модель оценки экономической целесообразности изготовления и эксплуатации технической системы по ведущему звену, предусматривающая определение минимальных затрат с учетом кратности работы деталей, наличие ремонта и замены последних в процессе эксплуатации технической системы. Разработан банк данных коэффициентов соответствия $K_{T}$, учитывающий влияние уровня технологических свойств на долговечность работы детали. Предложена методика определения ожидаемых затрат по верхнему и нижнему пределам с учетом кратности работы деталей, затрат на ремонт и их полную замену. Методика построена на сравнительной оценке критериальных параметров, отражающих технико-экономические показатели альтернативных технологий и материалов, путем выявления рациональных затрат на изготовление и эксплуатацию отдельно взятых деталей с учетом дополнительных затрат на их замену и кратность ремонта.

Ключевые слова: моральный и фризический износ, технологический цикл, коэффициент соответствия, экономические затраты и эффрективность, ведущее звено, инверсный режим, кратность работы, техническая система, простой, останов, оптимизационная модель, удельные затраты, автоматизация.

(C) Вайцехович С.М., Власов Ю.В.

Вайцехович Сергей Михайлович - канд. техн. наук, старший научный сотрудник, главный научный сотрудник ФГУП «Научно-производственное объединение „Техномаш“ имени С.А. Афанасьева», e-mail: ask-mlad@mail.ru.

Власов Юрий Вениаминович - канд. техн. наук, генеральный директор ФГУП «Научнопроизводственное объединение „Техномаш“ имени С.А. Афанасьева», e-mail: info@tmnpo.ru. 
Одним из важных направлений совершенствования конструкционных материалов является замена традиционных материалов на новые, в том числе материалы с конкретно заданными свойствами, а проблемы замены традиционных материалов на перспективные можно считать существенной частью общей проблемы отечественного управления эффективностью капитальных вложений и новой техники.

Сложившееся к настоящему времени отставание отечественного машиностроения по объемам производства новых конструкционных материалов от ведущих зарубежных государств обусловлено слабой экономической заинтересованностью изготовителя и потребителя, отсутствием научно обоснованной программы по разработкам, производству и применению новых материалов и изделий на них основе. В связи с нынешней ситуацией в экономике, либерализацией цен и рядом других факторов проработка и решение этих вопросов представляет практический интерес.

В существующих технико-экономических обоснованиях часто учитываются затраты, связанные с исследованием, изготовлением и внедрением новых материалов. Как правило, в них отсутствует сопоставление затрат на их создание и применение и не учитывается влияние технологических факторов на величину конечного экономического эффекта от использования нового. Расчеты нередко содержат методические ошибки, что не позволяет достоверно определить экономические преимущества. Это проявляется в условности выбора базы сравнения, расчетного года, приведении затрат в пропорции относительного изменения паспортной производительности техники, отрыве от системы ценообразования и оценки прогнозируемой прибыли. По этим причинам при использовании традиционных методов сравнительной эффективности капитальных вложений и новой техники реально объективные экономические расчеты крайне затруднены.

В настоящей работе предлагается принципиально новый подход к экономической модели расчета эффективности разработки и использования объектов потребления, учитывающий функциональное назначение материалов и потребительскую надежность изделий, сформулирована поставка и предложены пути решения задач: объединить интересы производителя и потребителя как совокупного объекта исследования.

Следует отметить, что для обеспечения корректности предлагаемых расчетов необходимо создать отраслевой банк данных, который содержит $[1,2]$ :

- зависимости физико-механических свойств деталей от технологии их получения;

- технологические переходы (ковка, штамповка, литье, механическая и термическая обработки), увязанные с экономическими показателями, затратами на изготовление, замену и ремонт деталей;

- экспериментальные номограммы и таблицы коэффициента соответствия $K_{\sigma}$, в котором в качестве исходных данных задаются: величина морального 
износа технической системы; технические характеристики материалов деталей; затраты на изготовление, замену и ремонт деталей, на остановы подсистем, узлов и связанные с ним простои технической системы в целом; коэффициенты соответствия временных параметров технологическим $K_{T}$ и экономических показателей техническим параметрам $K_{\sigma}$; фактическая стойкость деталей.

Соотношение между технологическими и эксплуатационными параметрами определяется коэффициентом соответствия $K_{T}$, равным тангенсу угла наклона траектории износа материала детали в процессе ее эксплуатации, так как затраты на изготовление детали технической системы пропорциональны ее эксплуатационной стойкости.

Соотношение между затратами и технологическими параметрами находят по коэффициенту соответствия $K_{\sigma}$, который в первом приближении выбирают из отраслевого банка данных, созданного на основе статистического анализа номенклатуры изделий, затем корректируют с помощью экспериментальных зависимостей, исследованных в настоящей работе и представленных в виде номограмм и таблиц. Коэффициент соответствия экономических показателей эксплуатационным параметрам $K_{N}$ определяется по универсальному экономическому критерию, который связывает технологические и эксплуатационные параметры с экономическими показателями [2].

Универсальный экономический критерий $\left(K_{T} \cdot \frac{K_{Z}}{K_{\sigma}}=\right.$ const) отражает следующую логику - в качестве исходных данных задаются: величина морального износа технической системы; технические характеристики материалов деталей; затраты на изготовление, замену и ремонт деталей, а также затраты на остановы подсистем и простои технической системы; коэффициенты соответствия временных параметров технологическим $\left(K_{T}\right)$ и экономических показателей техническим параметрам $\left(K_{\sigma}\right)$; фактическая стойкость деталей,

Задача моделирования направлена на установление между технологическими и эксплуатационными параметрами соотношений, соответствующих наименьшему уровню материальных затрат на изготовление и эксплуатацию деталей, входящих в техническую систему, при фиксированной величине долговечности последней.

Экономическая целесообразность использования того или иного материала определяется критерием долговечности детали относительно программной стойкости ведущего звена технической системы. Например, если при определении экономических показателей работы турбины колесо турбины принять за ведущее звено, то выбор материала для лопатки или вала должен проводиться таким образом, чтобы их эксплуатационные параметры не уступали аналогичной величине эксплуатационного параметра ведущего звена или являлись кратны его работе. 
Расчет целесообразности использования того или иного конструкционного материала для деталей машиностроения проводится следующим образом. Экономическая оценка затрат реализуется с помощью алгоритма, учитывающего функциональные особенности материалов в сфере эксплуатации непосредственно через эксплуатационные свойства деталей.

У потребителя [3, 4] экономическую эффективность получения изделий корректируют путем оптимизации технологического процесса, выявления «узких» мест в цепочке технологических операций [2].

Наряду с повышением качества изделий добиваются снижения трудозатрат. Здесь возрастающую роль играет снижение трудоемкости за счет более широкого внедрения в производственную практику автоматизированных и механизированных процессов во все звенья технологической цепочки. Для производителя $[5,6]$ предложен новый критериальный параметр автоматизации, учитывающий меру ручного труда в каждой отдельно взятой цепочке технологического маршрута, позволяющий оптимизировать затраты на изготовление деталей, стабилизировать повторяемость их физико-механических свойств на каждой операции [7].

Алгоритм расчета основывается на комплексном универсальном экономическом критерии $[2,8]$, учитывающем физико-механические свойства материалов, затраты на создание и изготовление изделий, во взаимосвязи с эксплуатационными параметрами последних. В модели принято, что величина затрат на изготовление и эксплуатацию изделий из конструкционных, материалов находится в прямой зависимости от технологических и эксплуатационных параметров, имеющих между собой устойчивую функциональную связь. Построение модели производится с учетом затрат на изготовление каждой детали технической системы, которые пропорциональны ее эксплуатационной стойкости - физическому (или моральному) износу $[9,10]$.

Время работы технической системы $[3,11,12]$ задается моральным износом $-t$. Количество замен по функциональному назначению одной и той же $i$-й детали за время работы технической системы определяется отношением

$$
P_{i}=\frac{t}{N_{i}}=A, B
$$

где $A$ - целое число; $B$ - дробная часть.

Учитывая, что регламентируемый запас прочности работы детали равен 1,05 , принимаем, что кратность работы $i$-й детали зависит от величины дробной части отношения $t$ к $N_{i}$ и определяется следующим образом:

$$
\begin{gathered}
\text { если } B<0,05 \text {, то } J_{i}=A \text {; } \\
\text { если } B \geq 0,05 \text {, то } J_{i}=A+1 \text {. }
\end{gathered}
$$

где $J_{i}-$ приведенная кратность работы $i$-й детали; 
Детали, для которых $J_{i} \leq 1$, могут работать без замены в течение всего периода морального износа $-t$; при $J_{i}>1$ - детали требуют замены.

Критерий надежности (эксплуатационной стойкости) работы деталей может быть определен из соотношения ((6) [4]), приведенного в первой части работы [2].

Необходимая и достаточная эксплуатационная стойкость деталей может быть определена из следующего уравнения:

$$
\left[g_{i}\right]=J_{i} \cdot\left(g_{i} \pm \Delta g_{i}\right),
$$

где $\Delta g_{i}$ - приращение до требуемой стойкости детали.

В более общем случае можно учесть различные коэффициенты запаса прочности для каждой детали, тогда

$$
\left[g_{i}\right]=\frac{g_{i}}{n_{i}},
$$

где $\left[g_{i}\right]$ - требуемая стойкость детали; $g_{i}-$ фактическая удельная стойкость $i$-й детали; $n_{i}-$ коэффициент надежности.

$$
0<g_{i}<\infty .
$$

Оптимальное значение $g_{\text {опт }}=1$.

Допустим, что $\pm \cdot \Delta g_{i}$ - показатель надежности работы детали, тогда $J_{i} \cdot \Delta g_{i}=G_{i}$, где $G_{i}$ - требуемая фактическая удельная стойкость детали.

Имеют место два взаимоисключающих случая.

Первый случай $\Delta g_{i}<0$

$$
\mid \begin{aligned}
& -\Delta g_{i}=\left(t_{i}-t^{*}\right)=f\left(J_{i}\right), \\
& +\Delta g_{i}=U\left(J_{i}\right)=0,
\end{aligned}
$$

где $f\left(J_{i}\right)$ - резерв эксплуатационной надежности $i$-й детали при заданной кратности $J$, при первой кратности $J_{i}=1$, удовлетворяющей моральный износ; $U\left(J_{i}\right)$ - величина эксплуатационной надежности, недостающей до заданной кратности $J_{i} ; t^{*}-$ заданное время морального износа работы (стойкость) детали; $t_{i}$ - текущий уровень эксплуатационной стойкости детали.

Второй случай $\Delta g_{i}>0$

$$
\mid \begin{aligned}
& -\Delta g_{i}=f\left(J_{i}\right)=0, \\
& +\Delta g_{i}=\left(t_{i}-t^{*}\right)=U\left(J_{i}\right)=0,
\end{aligned}
$$


В этом случае $f(1)>0$, а $U(1)=0$,

и при $J_{i}=1$

$$
f(1)=0, U(1)<0 \text {. }
$$

В случае оптимального значения $g_{\text {опт }}=g_{i}$

$$
g_{i}=\left[g_{i}\right]=1 \text {, }
$$

тогда отклонения $\pm \cdot \Delta g_{i}=0$

$$
f\left(J_{i}\right)=0, U\left(J_{i}\right)=0 .
$$

Третий случай $g_{i}>1$. Здесь $f_{1}(1)$ - избыток надежности при $J_{i}=1$; $\Pi_{i}(1)$ величина перерасхода материальных затрат; $Z_{i}=Z_{i}(1)-\Pi_{i}(1)-$ фактические затраты на изготовление $i$-й детали.

Физическая стойкость $i$-й детали $N_{i}$ превышает заданный моральный износ технической системы $t$, а именно $g_{i}>\left[g_{i}\right]$ на величину $f\left(J_{i}\right)$. Указанной величине соответствуют определенные материальные затраты на резерв эксплуатационной стойкости при $N_{i} \sim g_{i}$. Можно записать следующее выражение, соответствующее расчету по верхнему уровню затрат:

$$
\begin{gathered}
\left(Z_{i}\right)_{p}^{\prime}=F\left(f_{i}\right), \\
\left(Z_{i}\right)_{p}^{\prime}=Z_{i}+Z_{i}(1) \cdot\left(J_{i}-1\right)-\Pi\left(J_{i}\right)+Z_{3}\left(J_{1}-1\right) .
\end{gathered}
$$

Для случая, когда $J_{i}=1$, имеем $Z_{3}(1)=0$.

$$
\left(Z_{i}\right)_{p}^{\prime}=Z_{i}-\Pi_{i}(1)
$$

где $\left(Z_{i}\right)_{p}^{\prime}$ - рациональные затраты с учетом понижения уровня стойкости детали на $f\left(J_{i}\right) ; Z_{3}$ - затраты на замену детали (см. (25) [2]).

Поскольку $f\left(J_{i}\right)$ выражается конкретным числом и является частью $g_{i}$, поэтому можно записать

$$
\Pi_{i}\left(J_{i}\right)=f\left(J_{i}\right) \cdot K_{N_{i}},
$$

где $K_{N_{i}}-$ коэффициент соответствия для $i$-й детали. 
Учитывая, что имеет место соотношение

$$
\frac{\Pi_{i}\left(J_{i}\right)}{\Pi_{i}\left(J_{i}+1\right)}=\frac{f\left(J_{i}\right)}{f\left(J_{i}+1\right)}
$$

и принимая во внимание (8), для случая $g_{i}>1$ имеем

$$
K_{N_{i}}=\frac{\Pi_{i}\left(J_{i}\right)}{\Pi_{i}\left(J_{i}+1\right)},
$$

T.e. $K_{N_{i}}=\operatorname{tg} \alpha_{i}$.

Четвертый случай $0<g_{i}<1$. Физическая стойкость $i$-й детали $N_{i}$ недостаточна для достижения заданного уровня морального износа технической системы $t$, т.е. $g_{i}<\left[g_{i}\right]$ на величину $U\left(J_{i}\right)$.

Варианты расчета долговечности работы детали: $f_{i}$ - кратность использования $i$-й детали за период морального износа технической системы; $f_{i}(2)-$ избыток уровня надежности при $J_{i}=2 ; U_{i}(1)$ - недостающая величина уровня надежности для удовлетворения заданного периода морального износа работы технической системы; $Z_{i}$ - фактические затраты на изготовление и эксплуатацию $i$-й детали

Для увеличения надежности детали необходимо либо вложить дополнительные затраты $C_{i}\left(J_{i}\right)$, позволяющие повысить эксплуатационную стойкость, либо повысить кратность $J_{i}$ использования указанной $i$-й детали.

По аналогии с (7)при однократном использовании детали имеем:

$$
\begin{gathered}
J_{i}=1, \\
\left(Z_{i}\right)_{p}^{\prime \prime}=f\left(U_{i}\right), \\
\left(Z_{i}\right)_{p}^{\prime \prime}=Z_{i}+C_{i}(1)=Z_{i}(1),
\end{gathered}
$$

где $\left(Z_{i}\right)_{p}^{\prime \prime}$ - оптимальные затраты с учетом повышения уровня надежности деталей (см. (16) [2]).

С другой стороны, при увеличении кратности использования деталей уровень расходов будет меняться из (7) при $J_{i}=n$ :

$$
N_{p_{i}}=\sum_{i=1}^{n} N_{i}=t .
$$


Получается, что для удовлетворения морального износа количество деталей может быть увеличено до двух, $n=2$, для кратности $J=2$ из (6) будем иметь следующий уровень затрат:

$$
\left(Z_{i}\right)_{p}^{\prime}=Z_{i}+Z_{i}(1)-\Pi_{i}(2)=z_{i}(2) .
$$

Для принятия решения введем коэффициент пропорциональности, удовлетворяющий следующему соотношению:

$$
m_{i}=\frac{Z(1)}{Z(2)} .
$$

В случае, когда $m_{i}>1$, наиболее предпочтительным вариантом будет $Z(2)$ и наоборот. Например, в случае оценки затрат $\left(Z_{i}^{\min }\right)_{p}$ при нижнем пределе, величина дополнительных затрат $C_{i}(1)$ определяется простым суммированием затрат на детали, совокупность физических износов которых равна заданному уровню морального износа (7).

Коэффициент пропорциональности $m_{i}$ будет зависеть от значимости стоимостного параметра $\Pi_{i}\left(J_{i}\right)$.

Действительно, из (11) и (12) следует $Z(1)>Z(2)$.

Таким образом, $m_{i}$ показывает, целесообразно ли повышать технологические параметры детали с точки зрения вложения дополнительных затрат.

Рассмотрим случай условия, когда показатель кратности детали меньше морального износа $g_{i}<1$,

Имеем: $\operatorname{tg} \alpha_{i}=\frac{\sigma_{i}}{1-U_{i}}$.

Вариант № 1. Берем две детали с одинаковой стойкостью и получаем перебор по эксплуатационной стойкости $f_{i}(2)$.

$$
\left(Z_{i}\right)^{1}=2 \cdot Z_{i}+Z_{3}
$$

где $\left(Z_{i}\right)^{1}$ - затраты по варианту № $1 ; Z_{i}$ - затраты на одну деталь.

Вариант № 2. Повышаем физико-механические свойства детали $\sigma_{i}$ до $\sigma_{i}^{\max }$ по формуле $\left(\sigma_{i}^{\max }\right)^{\prime}=\operatorname{tg} \alpha_{i}$ при постоянном угле $\alpha_{i}$. Из уравнения (12) будем иметь

$$
\left(Z_{i}\right)^{2}=Z_{i}+K_{\sigma} \cdot\left[\left(\sigma_{i}^{\max }\right)-\sigma_{i}\right]
$$

где $\left(\sigma_{i}^{\max }\right)^{\prime \prime}$ - максимальная технологическая прочность. 
Вариант № 3. Уменьшаем угол $\alpha_{i}$, т.е. скорость физического износа детали $\operatorname{tg} \alpha_{i}=\sigma_{i}[4]$, имеем

$$
\left(Z_{i}\right)^{3}=Z_{i}+K_{\alpha} \cdot\left(\alpha_{i}-\alpha_{i}^{\prime}\right)
$$

где $K_{\alpha}$ - коэффициент соответствия скорости физического износа детали; $\alpha_{i}-$ исходный угол физического износа, соответствующий $\sigma_{i} ; \alpha_{i}^{\prime}-$ новый угол при сохранении $\sigma_{i}$.

Вариант № 4. Комбинируя варианты № 2 и 3, получим уравнение

$$
\left(Z_{i}\right)^{4}=Z_{i}+K_{\sigma} \cdot\left[\left(\sigma_{i}^{\max }\right)^{\prime}-\sigma_{i}\right]+K_{\alpha} \cdot\left(\alpha_{i}-\alpha_{i}^{\prime \prime}\right) .
$$

Поскольку конкретные численные значения $K_{\sigma}$ и $K_{\alpha}$ в литературных источниках отсутствуют и общеизвестно, что повышение уровня технологических свойств на несколько процентов требует увеличения материальных затрат, то согласно введенным обозначениям эффект выражается неравенством

$$
K_{\sigma} \cdot\left[\left(\sigma_{i}^{\max }\right)^{\prime}-\sigma_{i}\right] \gg Z_{i}, K_{\alpha} \cdot\left(\alpha_{i}-\alpha_{i}^{\prime \prime}\right) \gg Z_{i} .
$$

Таким образом, введенное предположение позволяет опустить рассмотрение вариантов № 2-4, что существенно упрощает выбор оптимального варианта. Однако исследование вариантов № 2-4 может привести к получению экономического эффекта в случае, если затраты на замену детали превышают стоимость ее изготовления.

Приведем к единому знаменателю дополнительные затраты $\Pi_{i}$ и $C_{i}$, представленные в уравнениях (8) и (11).

Рассмотрим варианты условия, когда $g_{i}>1$.

Вариант условия 1. Используется одна деталь $-\left(Z_{i}\right)^{1}=Z_{i}$. Величина избытка затрат имеет вид $\Pi_{i}(1)$, откуда рациональные затраты можно представить соотношением

$$
+\left(Z_{i}\right)_{p}^{1}=\left(Z_{i}\right)^{1}-\Pi_{i}(1)
$$

Вариант условия 2. Уменьшим перерасход затрат на производство детали путем понижения технологических параметров, например, изменением режима силовой обработки при получении детали методами обработки давлением или посредством варьирования режимов термообработки (закалка-нормализация) без изменения марки материала, из которого изготавливается деталь. Тогда $\sigma_{i}$ принимает значение $\left(\sigma_{i}^{\max }\right)^{\prime}$. 
При условии $\alpha_{i}=$ const будем иметь (11) и (12).

Отметим, что коэффициент соответствия $K_{\sigma}$ отражает долю приведенных затрат, обусловленных влиянием вида технологической обработки на изменение технологических параметров детали, а $K_{N}$ - долю приведенных затрат на изменение долговечности работы детали.

Решая совместно уравнения (11), (12) и (17), получаем зависимость

$$
K_{N}=K_{\sigma} \cdot\left(\sigma_{i}^{\min }\right)
$$

Используя банк данных значений $\left(\sigma_{i}^{\min }\right)$ для различных типов материалов и производственный опыт, можно построить указанную функциональную зависимость.

Поскольку для заданного вида материала, подвергнутого определенной технологической обработке, $\left(\sigma_{i}^{\min }\right)$ и $f_{i}(1)$ являются фиксированными величинами больше единицы, то с учетом (17)

$$
K_{N}=K_{\sigma} \cdot\left(\sigma_{i}^{\min }\right)
$$

которая представляет собой прямую линию, располагающуюся относительно координатных осей различным образом, в зависимости от постоянного коэффициента.

Построение графика функции

$$
K_{N}=\frac{K_{\sigma}}{t_{1}-t^{*}}\left[\sigma_{i}-\left(\sigma_{i}\right)^{\prime}\right]
$$

ведется следующим образом. Вначале исследуют функцию

$$
A_{i}=\frac{K_{\sigma}}{t_{1}-t^{*}} .
$$

При известных значениях $K_{\sigma}$ строят веерный график по (19) с учетом (20), в котором $t_{i}$ имеет фиксированное значение.

С другой стороны, при известных значениях $t_{i}$ можно построить зависимость (19) при фиксированных значениях

$$
K_{N}=\frac{K_{\sigma}}{t_{1}-t^{*}} \cdot \sigma_{i}^{*},
$$

где $\sigma_{i}^{*}=\left[\sigma_{i}-\left(\sigma_{i}\right)^{\prime}\right]$.

Таким образом, согласно зависимостям (20) и (21) коэффициент соответствия, учитывающий снижение долговечности работы детали за счет изменения 
технологии получения, будет определяться точкой на вогнутой поверхности параболоида для каждого конкретного класса материалов при заданных технологических параметрах.

Следовательно, для случая $g_{i}>1$ рациональные затраты на изготовление и эксплуатацию деталей с учетом (7), (8), (18) примут вид

$$
\left(Z_{i}\right)_{p}^{\prime}=Z_{i}-K_{N_{i}} \cdot f_{i}(1)
$$

Проведем аналогичное рассуждение для случая $g_{i}<1$. Рассмотрим влияние кратности на дополнительные затраты, повышающие стойкость по мере изменения технологической прочности

$$
K_{\sigma}\left[\left(\sigma_{i}^{\max }\right)-\sigma_{i}\right]
$$

и скорости физического износа

$$
K_{\alpha} \cdot\left(\alpha_{i}-\alpha_{i}^{\prime}\right)
$$

Случай, когда кратность $g_{i}>1$. Рациональные затраты на работу детали (11) с учетом ее замены:

$$
\left(Z_{i}\right)_{p}^{\prime \prime}=Z_{i}+Z\left(J_{i}\right)
$$

где $Z_{i}$ - затраты на изготовление $i$-й детали; $Z\left(J_{i}\right)$ - дополнительные затраты для $i$-й детали, при кратности $J_{i}>1$ с учетом (11) могут быть выражены:

$$
Z\left(J_{i}\right)=Z_{i}(1) \cdot\left(J_{i}-1\right)+C\left(J_{i}\right)+Z_{3} \cdot\left(J_{i}-1\right) .
$$

В то же время из формулы (12) [4] имеем

$$
C\left(J_{i}\right)=Z_{i} \cdot\left(e^{K_{N} \cdot U_{i}}-1\right)+Z_{i} \cdot\left(J_{i}^{*}-J_{i}\right),
$$

где $Z_{i}(1)$ - затраты для $i$-й детали первой кратности (фактические затраты);

$$
Z_{i}=Z_{i}(1), n \cdot Z_{i}(1)=Z_{i}(n)
$$

$Z_{3}$ - дополнительные затраты на замену деталей; $K_{N_{i}}$ - коэффициент соответствия затрат эксплуатационным параметрам детали согласно (19),

$$
K_{N_{i}}=\frac{K_{\sigma_{i}}}{K_{T_{i}}},
$$

$K_{\sigma_{i}}$ - коэффициент соответствия затрат технологическим параметрам, выбирается из таблицы;

$K_{i}=K_{\sigma_{i}} ; J_{i}^{*}-$ предельная кратность $i$-й детали, при которой происходит переход от недостатка эксплуатационной стойкости к ее избытку: 


$$
U\left(J_{i}\right)=U_{i}(1)-g_{i}\left(J_{i}-1\right)
$$

Для переходной зоны на рубеже от $U\left(J_{i}\right) \rightarrow f\left(J_{i}\right), J_{i} \rightarrow J_{i}^{*}$.

$$
U\left(J_{i}\right)=f\left(J_{i}\right)=g_{i}\left(J_{i}^{*}-J_{i}\right)-f_{i}\left(J_{i}^{*}\right) .
$$

где $f_{i}\left(J_{i}^{*}\right)$ - первое положительное значение параметра надежности при переходе от $U\left(J_{i}\right)$ к $f\left(J_{i}\right)$; соответствующей кратности $J_{i}^{*}$ при $J_{i} \geq J_{i}^{*}$ происходит переход в расчетах от $C\left(J_{i}\right)$ к П $\left(J_{i}\right)$,

$$
C\left(J_{i}\right) \Pi\left(J_{i}\right) .
$$

Проанализировав (22)-(29), приведем общее уравнение для расчета затрат на изготовление $i$-й детали, с учетом дополнительных вложений и соответствующей кратности:

$$
\left(Z_{i}\right)_{p}^{\prime \prime}=Z_{i}+Z_{i}\left(J_{i}-1\right)+C\left(J_{i}\right)+Z_{3}\left(J_{i}-1\right) .
$$

После упрощения имеем

$$
\left(Z_{i}\right)_{p}^{\prime \prime}=Z_{i} \cdot J_{i}+C\left(J_{i}\right)+Z_{3}\left(J_{i}-1\right) .
$$

При $U\left(J_{i}\right)=f\left(J_{i}\right)=0$ - величины дополнительных затрат также равны нулю, т.е. $C\left(J_{i}\right)=0$ и $\Pi\left(J_{i}\right)=0$. Тогда

$$
\left(Z_{p}\right)_{p}=Z_{i} \cdot J_{i}+Z_{3}\left(J_{i}-1\right)
$$

Как изложено выше, для оценки экономической целесообразности изготовления и эксплуатации технической системы по предложенной методике необходимо создание банка данных, где должны быть отражены следующие позиции [4]:

- область изменения физико-механических свойств материала;

- технологичность материала;

- затраты на технологические переделы изготовления деталей;

- отечественные и зарубежные аналоги материалов и изделий на их основе;

- коэффициенты $K_{T}$ и $K_{\sigma}$, учитывающие изменение величин затрат от технологических и эксплуатационных параметров.

Наличие указанного банка данных позволит существенно сократить время поиска наиболее экономичных подходов к решению технических задач в различных отраслях народного хозяйства. 
Разработанная методика опробована в энергетическом машиностроении для деталей привода СУЗ ШЭМ-3 водо-водяного энергетического реактора ВВЭР-1000.

В качестве исходных данных задается величина морального износа технической системы [13], технические характеристики материалов и входящих в нее деталей, величина их затрат, затраты на замену деталей и ремонт, а также на остановы блоков и простои технической системы.

В разработанной модели принято, что величина прогнозируемых затрат на изготовление и эксплуатацию новых конструкционных материалов находится в прямой зависимости от технологических и эксплуатационных параметров, имеющих между собой устойчивую функциональную связь, однозначно определяемую по всему диапазону возможных изменений технологических факторов.

Задачей моделирования является установление соотношений между технологическими и эксплуатационными параметрами, соответствующих меньшему уровню материальных затрат на изготовление и эксплуатацию деталей, входящих в техническую систему при заданной величине долговечности последней. Соотношение между технологическими и эксплуатационными параметрами определяется коэффициентом соответствия $K_{T}$, равным тангенсу угла наклона траектории износа материала детали в процессе ее эксплуатации [4].

Коэффициент соответствия $K_{T}$ задается технологическими параметрами в соответствии с классом используемого материала $[14,15]$, коэффициент соответствия $K_{\sigma}$ берется из банка данных, экспериментальных номограмм и таблиц, разница времен морального износа и фактической стойкости детали технической характеристикой материала.

Коэффициент соответствия $K_{N}$ определяется из универсального экономического соотношения (21) и позволяет при экономическом анализе технических систем пользоваться только технологическими и временными зависимостями для отдельных деталей, входящих в техническую систему.

Алгоритм построения модели оптимизации затрат сводится к следующему: оптимизацию затрат технической системы проводят с помощью моделирования единичных затрат на каждую деталь по ведущему звену. За ведущее звено принимается последовательно каждая деталь технической системы вне зависимости от значения ее стойкостных и других параметров. При этом к кратности ведущего звена приводятся кратности всех остальных деталей узла, определяются затраты по кратности работы деталей, что позволяет выявить минимальные затраты, расходуемые на всю техническую систему.

При $J_{i}>1$ учитываются следующие соотношения:

$$
G_{i}=\frac{N_{i}}{t} \cdot J_{i},
$$


если $G_{i}>1$, то

$$
U\left(J_{i}\right)=0, f\left(J_{i}\right)=\left(g_{i} \cdot J_{i}\right)-1 .
$$

если $G_{i}<1$, то

$$
U\left(J_{i}\right)=1-\left(g_{i} \cdot J_{i}\right), \quad f\left(J_{i}\right)=0,
$$

если $G_{i}=1$, то

$$
U\left(J_{i}\right)=0, f\left(J_{i}\right)=0,
$$

т.е. дополнительные затраты для кратности $J_{i}$ равны нулю.

$C\left(J_{i}\right)=0 ; \Pi\left(J_{i}\right)=0$ и затраты $\left(Z_{i}\right)_{p}$ вычисляются по (28).

При $J_{i} \geq J_{i}^{*}$ имеем переход от затрат $C\left(J_{i}\right)$ к затратам П $\left(J_{i}\right)$.

Рациональные затраты на изготовление и эксплуатацию деталей с учетом (6) и (28) выразим наиболее общим соотношением

$$
\left(Z_{i}\right)_{p}=Z_{i}+Z_{i} \cdot\left(J_{i}\right)
$$

с учетом (23) имеем уравнения расчета дополнительных затрат для деталей произвольной кратности

$$
Z_{i}\left(J_{i}\right)=Z_{i}(1) \cdot\left(J_{i}-1\right)+\left[C\left(J_{i}\right)-\Pi\left(J_{i}\right)\right]+Z_{3}\left(J_{i}-1\right) .
$$

Из опыта промышленного освоения новых конструкционных материалов следует, что дополнительные затраты $C\left(J_{i}\right)$, направленные на увеличение стойкости детали от $U\left(J_{i}\right)$ до $f\left(J_{i}\right)$ при сохранении заданной нормативной кратности $J_{i}=$ const, находятся в пределах дополнительных затрат переходного значения $J^{*}$ без учета затрат на перерасход эксплуатационной стойкости $\Pi\left(J_{i}\right)$ и являются его нижней границей.

$$
C_{i}^{\min }\left(U_{i}\right)=Z_{i}\left(J_{i}^{*}-J_{i}\right)+Z_{3}\left(J_{i}^{*}-J_{i}\right) .
$$

Выражение (23) с учетом (35) и после соответствующих упрощений примет вид

$$
Z_{i}^{\min }\left(J_{i}\right)=Z_{i}\left(J_{i}^{*}-J_{i}\right)+C_{i}^{\min }\left(U_{i}\right)+Z_{3}\left(J_{i}-1\right) .
$$

Отмеченное можно проиллюстрировать на примере привода ШЭМ, состоящего из пяти деталей. Для детали № 5: 


$$
\begin{gathered}
C_{5}^{\min }\left(U_{i}\right)=Z_{5}\left(J_{i}^{*}-J_{i}\right)+Z_{3}\left(J_{i}^{*}-J_{i}\right), \\
Z_{5}^{\min }\left(J_{i}\right)=Z_{5}\left(J_{i}^{*}-J_{i}\right)+C_{5}^{\min }\left(U_{i}\right)+Z_{3}\left(J_{i}-1\right) .
\end{gathered}
$$

Затраты на привод ШЭМ складываются из суммы минимальных затрат по отдельно взятым деталям с учетом их возможного частичного ремонта и затрат на замену.

Рациональное количество деталей, подлежащих изготовлению, с учетом возможного ремонта и последующего использования, можно выбрать по величине кратности $J_{i}^{*}$ :

$$
n=J_{i}^{*}
$$

При известных затратах на ремонт и заданных значениях $K$ и $r$ оптимальное количество деталей уточняется по источнику [4, формула (25)]:

$$
Z_{3}=r \cdot Z_{\text {д }}^{1} \cdot(n-1) .
$$

Если кратности $J_{i}^{*}$ предшествует кратность, при которой $U\left(J_{i}\right)=0$ и $f\left(J_{i}\right)=0$, то нужное количество деталей для удовлетворения морального износа привода можно принять равным величине предшествующей кратности

$$
\left(J_{i}^{*}-1\right)
$$

При оптимизации затрат технической системы рассматривают затраты на отдельно взятые детали, входящие в нее [16], с учетом варьирования кратностей деталей и последующего сопоставления выявленных затрат между собой [17].

Учет ремонта деталей, кратность которых определяется соотношением $J_{i}^{*} \gg 3$, позволяет снизить затраты на изготовление и эксплуатацию технической системы [18]. В ряде случаев затраты на ремонт детали меньше, чем затраты на изготовление новой детали:

$$
Z_{i}^{p}<Z_{i}
$$

Расчетные параметры выражены общим уравнением

$$
Z(N, A)=A \cdot\left(Z_{g}+\sum_{i+1}^{y} Z_{i}^{p}\right)+\chi \cdot Z_{j}^{p},
$$

где $N$ - количество деталей, входящих в техническую систему; $A$ - количество деталей, изготавливаемых заново; $Z_{i}^{p}$ - затраты на ремонт $i$-й кратности, $i=$ 
$=1,2,3 ; Z_{g}$ - затраты на изготовление новых деталей; $\chi$ - остаток от деления $\frac{N}{A} ; y$ - целая часть от $\left(\frac{N}{A}-1\right) ; y=\mathrm{I}, 1,2,3 \ldots j=\frac{N-1}{A}$.

Для апробации предложенного метода расчета затрат у потребителя решена задача оптимизации затрат на эксплуатацию привода ШЭМ, путем моделирования кратности ремонта, величины общего количества ремонта и количества заново изготавливаемых деталей.

В качестве примера рассмотрим деталь № 4 привода ШЭМ, физический износ которой составляет 5 лет. Известно, что время морального износа ВВЭР-1000 составляет 40 лет. Поэтому общее количество деталей № 4, необходимое для удовлетворения морального износа реактора, будет равно кратности работы указанной детали, т.е. восьми [19].

Если иметь в виду, что производитель изготавливает для одного комплекта привода ШЭМ одну деталь № 4, то, следовательно, ее ремонт берет на себя потребитель. По кратности работы детали № 4 видно, что в процессе эксплуатации реактора ее необходимо будет ремонтировать семь раз. При этом к затратам на замену детали и простои подсистемы (привода ШЭМ) прибавляются затраты, связанные с ремонтом детали. Время ремонта детали входит во время останова привода [20].

Для сокращения затрат на останов привода ШЭМ целесообразно исключить время, приходящееся на ремонт детали. Из статистического анализа затрат на простой технической системы следует, что для деталей, имеющих кратность работы три и более раз, наиболее рациональным является изготовление по меньшей мере двух деталей. В этом случае время на ремонт детали не входит в общее время затрат на останов подсистемы.

Таким образом, от того, какое количество деталей будет изготовлено заново, а какое подвергнуто тому или иному ремонту определенной кратности, будут зависеть общие затраты потребителя.

В качестве исходных данных для решения задачи оптимизации затрат на ремонт выступали следующие затраты: изготовление детали $\left(Z_{A}\right)$, замену $\left(Z_{3}\right)$, ремонт $\left(Z_{i}^{p}\right)$, в соответствии с кратностью ремонта $\left(Z_{1}^{p}, Z_{2}^{p}, Z_{3}^{p}\right.$, и т.д.), а также количество остановов привода ШЭМ.

Поставим задачу: физический износ детали № 4 привода ШЭМ ВВЭР-1000 по статистическим данным составляет 5 лет. Моральный износ ВВЭР-1000 рассчитан на 40 лет. Таким образом, кратность детали № 4 по (1), (2) составляет:

$$
J_{1}=\frac{40}{5}
$$




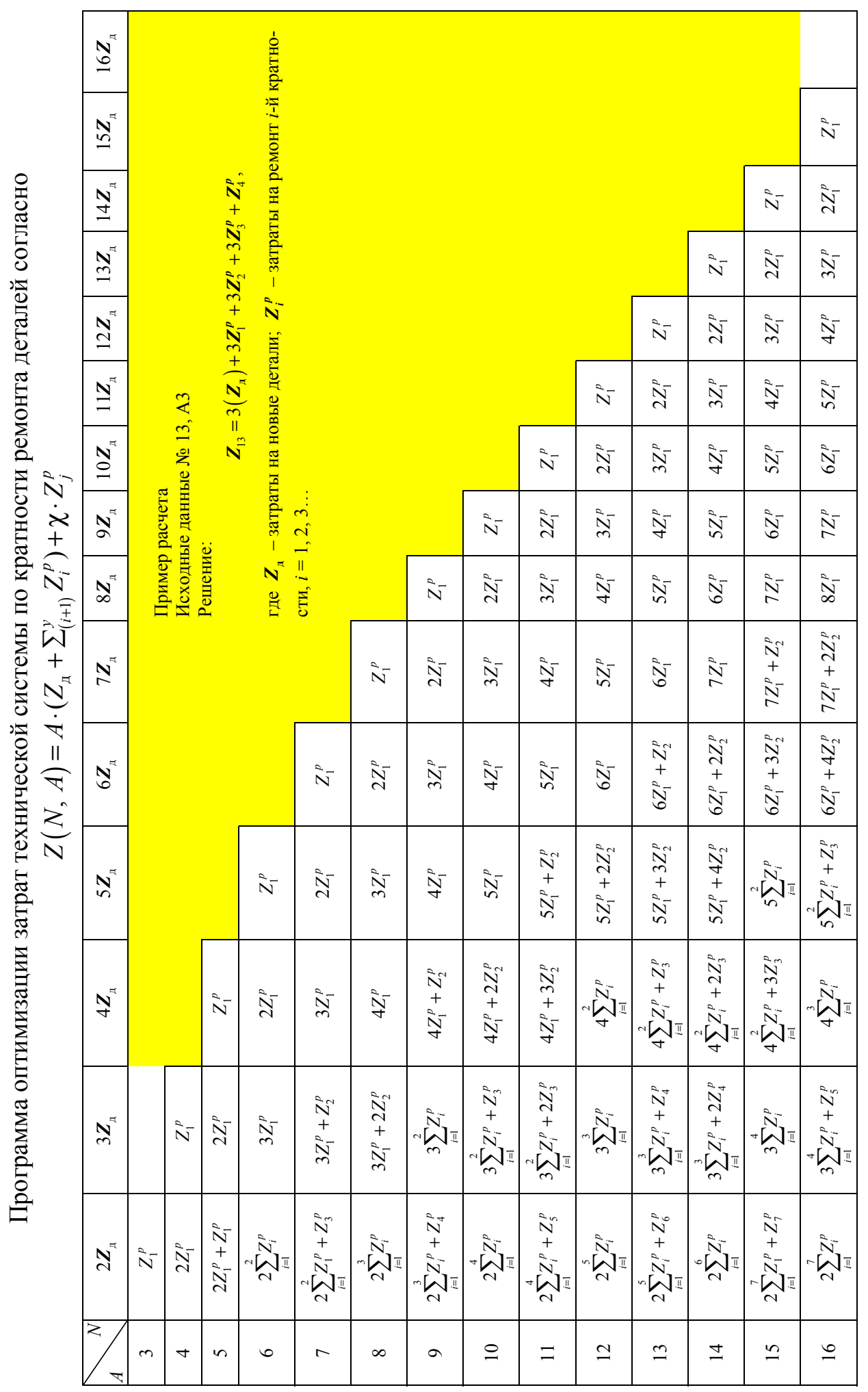


Следует определить, какое количество деталей привода за весь период эксплуатации ВВЭР-1000 необходимо изготовить заново [19] и сколько использовать после ремонта с учетом его кратности. Моделирование проводим изменением параметров $K, Z_{i}^{p}$.

Предположим, что затраты на замену детали ниже стоимости ремонта не менее чем на порядок, в то же время затраты на ремонт ниже затрат на изготовление новой детали

$$
Z_{3} \ll Z_{i}^{p} ; \quad Z_{i}^{p}<Z_{f}
$$

При этом $Z_{1}^{p}<Z_{2}^{p}<Z_{n}^{p}<Z_{n=1}^{p}$, т.е. затраты на ремонт по мере роста кратности ремонта возрастают, а величина затрат на замену деталей во всех случаях постоянна:

$$
Z_{3}=\text { const. }
$$

Положим затраты на изготовление детали равны $Z_{\text {д }}$, затраты на ремонт $i$-й детали $-Z_{i}^{p}$.

Рассмотрим несколько возможных вариантов с учетом (37).

Bapuaнm № 1. Изготавливают восемь деталей № 4. По мере износа деталь меняют на новую, а отработанную деталь отправляют на металлолом. Таким образом, общие затраты на изготовление и эксплуатацию $\left(Z_{\mathrm{o}}\right)^{1}=8 \cdot Z_{\text {д }}$.

Bapuaнm № 2. Изготавливают две детали, которые последовательно ремонтируют и заменяют по мере их выхода из строя. Расчет количества ремонта и величин кратности определяется по (37).

Обозначим затраты на первый ремонт $-Z_{1}^{p}$, второй $-Z_{2}^{p}$, третий $-Z_{3}^{p}$.

При этом затраты на ремонт по мере увеличения кратности ремонта возрастают, т.е. $Z_{1}^{p}<Z_{2}^{p}<Z_{3}^{p}$.

Общие затраты $\left(Z_{0}\right)^{2}=2 Z_{\text {д }}+2 Z_{2}^{p}$.

Bapuaнm № 3. Изготавливают три детали. Ремонт первой кратности составляет $3 Z_{1}^{p}$; ремонт второй кратности $-2 Z_{2}^{p}$.

Общие затраты $\left(Z_{\mathrm{o}}\right)^{3}=3 Z_{\text {д }}+3 Z_{1}^{p}+2 Z_{2}^{p}$.

Bapuaнm № 4. Изготавливают четыре детали, ремонт первой кратности $-4 Z_{1}^{p}$.

Общие затраты $\left(Z_{\mathrm{o}}\right)^{4}=4 Z_{\text {д }}+4 Z_{1}^{p}$.

Bapuaнm № 5. Изготавливают пять деталей, ремонт первой кратности $3 Z_{1}^{p}$. Общие затраты $\left(Z_{\mathrm{o}}\right)^{5}=5 Z_{\text {д }}+3 Z_{1}^{p}$.

И далее по аналогии. 
Вариант № 6: $\left(Z_{\mathrm{o}}\right)^{6}=6 Z_{\text {д }}+2 Z_{1}^{p}$.

Вариант № 7: $\left(Z_{\mathrm{o}}\right)^{7}=7 Z_{\text {д }}+Z_{1}^{p}$.

Для определенности рассмотрим две версии затрат.

Первая версия:

$$
Z_{1}^{p}=\frac{1}{2} \cdot Z_{\text {д }} ; \quad Z_{2}^{p}=\frac{2}{3} \cdot Z_{\text {д }} ; \quad Z_{3}^{p}>Z_{2}^{p} .
$$

Тогда общие затраты по каждому варианту составят:

$$
\begin{gathered}
\left(Z_{\mathrm{o}}\right)^{1}=8,0 Z_{\text {д }} ;\left(Z_{\mathrm{o}}\right)^{3}=5,8 Z_{\text {д }} ;\left(Z_{\mathrm{o}}\right)^{5}=6,5 Z_{\text {д }} ; \\
\left(Z_{\mathrm{o}}\right)^{2}=6,0 Z_{\text {д }} ;\left(Z_{\mathrm{o}}\right)^{4}=6,0 Z_{\text {д }} ;\left(Z_{\mathrm{o}}\right)^{6}=7,0 Z_{\text {д }} ;\left(Z_{\mathrm{o}}\right)^{7}=7,5 Z_{\text {д }} .
\end{gathered}
$$

Из принятых предположений следует, что из рассмотренных вариантов первой версии наиболее экономичным является вариант № 3. Заслуживает внимания тот факт, что для данного вывода не требуется точного числового значения затрат на ремонт третьей кратности $Z_{3}^{p}$, а достаточно лишь его нижней оценки $Z_{3}^{p} \geq Z_{2}^{p}$.

\section{Вторая версия:}

$$
Z_{1}^{p}=\frac{1}{3} \cdot Z_{\text {д }} ; \quad Z_{2}^{p}=\frac{1}{2} \cdot Z_{\text {д }} ; \quad Z_{3}^{p}>\frac{2}{3} \cdot Z_{2}^{p} .
$$

Общие затраты по каждому варианту:

$$
\begin{gathered}
\left(Z_{\mathrm{o}}\right)^{1}=8,0 Z_{\text {д }} ;\left(Z_{\mathrm{o}}\right)^{3}=5,0 Z_{\text {д }} ;\left(Z_{\mathrm{o}}\right)^{5}=6,0 Z_{\text {д }} ; \\
\left(Z_{\mathrm{o}}\right)^{2}=5,0 Z_{\text {д }} ;\left(Z_{\mathrm{o}}\right)^{4}=5,3 Z_{\text {д }} ;\left(Z_{\mathrm{o}}\right)^{6}=6,6 Z_{\text {д }} ;\left(Z_{\mathrm{o}}\right)^{7}=7,3 Z_{\text {д }} .
\end{gathered}
$$

Из уравнений общих затрат на производство и эксплуатацию деталей (6) и (28) выведем уравнения для определения величин материальных затрат отдельно как у производителя, так и у потребителя. Это необходимо для выявления их доли в целях возможной корректировки в сторону уменьшения.

В случае если $U_{i}\left(J_{i}\right)=0$ и $f_{i}\left(J_{i}\right)>0$, имеем

$$
\left(Z_{i}\right)_{p}^{\mathrm{\Pi}}=Z_{i}-\frac{K_{N_{i}} \cdot f\left(J_{i}\right)}{J_{i}} .
$$

В случае если $U_{i}\left(J_{i}\right)>0$ и $f_{i}\left(J_{i}\right)=0$, имеем 


$$
\left(Z_{i}\right)_{p}^{\Pi}=Z_{i} \cdot \frac{1}{J_{i}} \cdot\left(e^{K_{N} \cdot U_{i}}+J^{*}-1\right),
$$

где $\left(Z_{i}\right)_{p}^{\text {п }}$ - затраты у производителя на изготовление $i$-й детали.

Для обоих случаев затраты у потребителя на эксплуатацию $i$-й детали за период морального износа технической системы составят:

$$
\left(Z_{i}\right)_{p}^{\ni}=\left(Z_{i}\right)_{p}^{\Pi} \cdot\left(J_{i}-1\right)+Z_{3} \cdot\left(J^{*}-1\right) .
$$

Долю материальных затрат, приходящихся на период эксплуатации деталей, можно определить следующим образом:

$$
\mathrm{M}=\frac{\left(Z_{i}\right)_{p}^{\ni} \cdot 100 \%}{Z_{\mathrm{o}}}
$$

где $Z_{\text {о }}=\left(Z_{i}\right)_{p}^{\Pi}+\left(Z_{i}\right)_{p}^{э}$ - общая сумма затрат на изготовление и эксплуатацию деталей за период морального износа технической системы.

Итогом расчета рациональных затрат на изготовление и эксплуатацию деталей технической системы из новых конструкционных материалов является построение сетевого графика, определение количества остановов подсистем и простоев технической системы, их оптимизация и расчет общих затрат на техническую систему.

Рассмотренный алгоритм в виде комплекса программ в режиме диалога. Расчетная информация представляется в виде сетевых графиков и таблиц. Время расчета одного варианта - 10-15 мин.

В качестве критерия экономической оценки эффективности производства вводится коэффициент автоматизации как для всего техпроцесса в целом, так и для отдельной операции.

Для оценки степени автоматизации предлагаются следующие формулы:

$$
\bar{K}=K_{1} \cdot \frac{t_{1}}{t_{\mathrm{o}}}+K_{21} \cdot \frac{t_{2}}{t_{\mathrm{o}}}+\ldots+K_{n} \cdot \frac{t_{n}}{t_{\mathrm{o}}}=\frac{1}{t_{\mathrm{o}}} \cdot \sum_{i=1}^{n} K_{i} \cdot t_{i},
$$

где $K_{i}$ - степень автоматизации на $i$-й операции; $i=1,2, \ldots n ; t_{i}-$ время $i$-й операции; $t_{\mathrm{o}}=\sum_{i=1}^{n} t_{i}-$ общее время техпроцесса.

Коэффициент автоматизации для каждого вида операции определяется из отношения

$$
K_{i}=\frac{\Pi_{\text {т }}}{\Pi_{\text {пр }}},
$$

где $\Pi_{\text {т }} ; \Pi_{\text {пр }}-$ соответственно теоретический и практический параметры автоматизации. 
Под теоретическим параметром автоматизации для данной операции понимается величина предельного значения выбранного параметра (себестоимость, время операции и др.), которая достигается при соблюдении современных технологических требований. Под практическим параметром автоматизации выступают используемые на практике нормативы требований.

В качестве примера выбора прогрессивной технологии при производстве деталей корпуса атомного реактора ВВЭР-1000 - обечайки с патрубком - взяты альтернативные варианты, включающие следующие переделы:

$K_{1}$ - кузнечная обработка; $K_{2}$ - механическая обработка; $K_{3}$ - сварка.

Вариант № 1. Гладкие обечайки со сварными патрубками.

Пусть $K_{1}=0,1 ; K_{2}=0,8 ; K_{3}=1$;

$$
t_{1}=1 ; t_{2}=1 ; t_{3}=8
$$

Следовательно, $t_{\mathrm{o}}=10$ (условная единица измерения времени).

Тогда $\bar{K}=\frac{1}{10} \cdot(0,1 \cdot 1+0,8 \cdot 1+1 \cdot 8)=0,89$.

Bариант № 2. Обечайки с выштампованными патрубками.

Пусть $K_{1}=1 ; K_{2}=1 ; K_{3}=0,9$;

$$
t_{1}=5 ; t_{2}=10 ; t_{3}=1 \text {. }
$$

Следовательно, $t_{\mathrm{o}}=16$,

тогда $\bar{K}=\frac{1}{16} \cdot(1 \cdot 5+1 \cdot 10+0,9 \cdot 1)=0,99375$.

Введем понятие дисперсии Д, характеризующее разброс степени автоматизации по операциям.

$$
\text { Д }>0 .
$$

В идеале Д $=0$ и $\bar{K}=1$, это может быть, если все $K_{i}=1$.

Если хотя бы одно $K_{i} \neq 1$, то Д $>0$ и $\bar{K}<1$.

$$
\text { Д }=\frac{n}{n-1} \cdot \frac{\sum_{i=1}^{n} t_{i} \cdot\left(K_{i}-\bar{K}\right)^{2}}{t_{0}}
$$

или

$$
\text { Д }=\frac{n}{n-1} \cdot \frac{\sum_{i=1}^{n} t_{i} \cdot K_{i}^{2}}{t_{0}}-(\bar{K})^{2} .
$$

Для первого варианта имеем $Д_{1}=0,109$. 
Для второго варианта имеем $Д_{2}=0,001$.

Если для сравниваемых техпроцессов коэффициенты автоматизации незначительно отличаются друг от друга, то для характеристики рассеивания степени автоматизации по операциям следует принимать относительную характеристику рассеивания: $N_{v}$ - коэффициент вариации - отношение среднего квадратичного отклонения $\sigma=\sqrt{Д}$ в процентах к среднему $\bar{K}$ :

$$
N_{v}=\frac{\sigma}{\bar{K}} \cdot 100 \% \text {. }
$$

Для первого варианта $N_{v_{1}}=37 \%$.

Для второго варианта $N_{v_{2}}=3 \%$.

Таким образом, второй вариант является предпочтительным по степени автоматизации.

Предложенный коэффициент автоматизации и его математическое выражение в качестве критерия экономической эффективности у производителя позволяет выявить наиболее рациональную технологию.

Предложенный коэффициент автоматизации и его математическое выражение в качестве критерия экономической эффективности у производителя позволяет выявить наиболее рациональную технологию.

В работе созданы пакеты программ, охватывающие все стороны рассматриваемого вопроса. В частности, программы выбора оптимальной кратности работы деталей по минимальным затратам; выбора кратности ремонта с учетом затрат на замену деталей; построение коэффициента соответствия $K_{\sigma}$ в зависимости от затрат на технологическую обработку детали и другие штатные подпрограммы, позволяющие оптимизировать затраты на изготовление и эксплуатацию технической системы.

\section{Bbыводbl:}

1. Для оценки экономической эффективности технологических процессов у производителя разработан критериальный параметр, включающий в себя коэффициент автоматизации, дисперсию, коэффициент вариации, позволяющий выявить эффективную технологию; у потребителя - кратность работы деталей, учет ремонта и замены вышедшей из строя детали. Критериальный параметр позволяет при сравнении альтернативных технологий выявить наиболее рациональную, с точки зрения стабилизации уровня физико-механических свойств материалов на технологических переделах.

2. На основании экспериментальных данных освоения новых конструкционных материалов в энергомашиностроении методом математического моделирования построены таблицы расчета коэффициента соответствия $K_{\sigma}$, учитывающего изменение затрат от уровня технологических свойств детали. 
3. Разработан универсальный экономический критерий $K_{t} \cdot \frac{K_{N}}{K_{\sigma}}=$ const, поЗволяющий при экономическом анализе технической системы пользоваться только технологическими и временными зависимостями отдельных деталей, входящих в рассматриваемую техническую систему.

5. Разработан банк данных коэффициентов соответствия $K_{t}$, учитывающий влияние уровня технологических свойств на долговечность работы детали.

\section{Список литературы}

1. Вайцехович С.М., Кужель А.Е. Комплексная система оценки применения конструкционных материалов в промышленности // Вестник машиностроения. - 1997. - № 4. - С. 29-32.

2. Вайцехович С.М., Власов Ю.В. Комплексная система оценки эффективности производства и потребления продукции машиностроения // Вестник Пермского национального исследовательского политехнического университета. Социально-экономические науки. - 2020. - № 2. - С. 146-167.

3. Повышение долговечности автомобильных металлических материалов / Г.П. Гуслякова, С.И. Жбанников [и др.]. - Нижний Новгород, 1991. - 64 с.

4. Ласковый А.А. Методические рекомендации по комплексной оценке эффективности машиностроительного производства в НПО. - Киев: Наукова думка, 1982. -121 с.

5. Васильев Г.А. Планирование повышении экономической эффективности машиностроительного производства. - М.: Машиностроение: 1985. - 207 с.

6. Голосовский С.И., Никитин А.П., Тутов И.Е. Технико-экономическая эффективность применения перлитных сталей в энергомашиностроении // Экономическая эффективность внедрения новых технологических процессов и конструкционных материалов в тяжелом машиностроении / ЦБТИ. - М., 1961. - С. 93-97.

7. Маленкович В.Б., Кужель А.Е. Новые методы оценки эффективности технологических процессов в машиностроении // Технология, экономика, организация производства и управления: экспресс-информ. / ЦНИИТмаш. - М., 1991. - 18-1-91-03. - С. 1-7.

8. Методические положения по выбору параметров, наиболее полно характеризующих потребительские качества продукции и связанные с затратами материальных и топливо-энергетических ресурсов / Госплан СССР, НИИПиН. M., 1981. -32 c.

9. Ерохина Л.С., Калугина К.В., Михайлов С.К. Методы прогнозирования развития конструкционных материалов. - Л.: Машиностроение, 1980. - 256 с.

10. Иванов Ю.Н., Токарев В.В. Оценивание эффективности новых технологий по межотраслевым моделям // Модели и методы в прогнозировании научно-технического прогресса / ВНИИСИ. - М., 1984. - Вып. 2. - С. 4-18. 
11. Иванов А.В., Иванова В.А. Формирование и обновление систем изделий. - М.: Экономика, 1981. - 56 с.

12. Колесниченко Р.А. Прогрессивные технологии получения заготовок и экономически целесообразные границы их применения // Экономика машиностроения / МЭ АН УССР. - Киев, 1981. - С. 59-63.

13. Методические рекомендации по комплексной оценке эффективности мероприятий, направленных на ускорение научно-технического прогресса / ГКНТ СССР. - М., 1988. - № 60/52. - 120 c.

14. Пашко И.Г., Павлов В.И., Спектор А.Н. Экономический эффект новых конструкционных материалов. - М.: Знание, 1987. - 64 с.

15. Поляк А.М. Повышение качества и улучшение использования металлов / МИСиС. - М., 1980. - 51 с.

16. Садовничая С.Г. Создание нормативов производственных затрат на новую продукцию // Экономические проблемы сокращения цикла процесса «исследование-разработка-производство»: тез. докл. науч.-практ. конф. Днепропетровск, 1987. - С. 178-179.

17. Савоськин М.Е. Снижение металлоемкости изделий путем применения новых материалов и технологий. - Киев: Наукова думка, 1980. - 11 с.

18. Маленкович В.Б., Кужель А.Е. Исследование взаимосвязи эксплуатационных параметров новых конструкционных материалов с экономическими показателями // Синергетика. Новые технологии получения и свойства металлических материалов: материалы 2 Всесоюз. симп. / Ин-т металлургии и материаловедения им. А.А. Байкова. - М., 1991. - 176 с.

19. Филипцев Е.Я., Данилин В.И. Модель определения сроков замены изделий новыми при разработке пятилетнего плана объединения // Экономикоматематические модели в системе управления предприятием / под ред. Н.П. Федоренко и И.П. Шубкиной. - М.: Машиностроение, 1983. - С. 148-160.

20. Терехин В.И. Экономическое обоснование обновления продукции // Экономические проблемы межотраслевого и регионального управления научно-техническим прогрессом / ЛИЭИ. - Л., 1986. - С. 90-99.

\section{References}

1. Vaitsekhovich S.M., Kuzhel' A.E. Kompleksnaia sistema otsenki primeneniia konstruktsionnykh materialov $\mathrm{v}$ promyshlennosti [The complex evaluation system of construction materials application in industrial production]. Vestnik mashinostroeniia, 1997, no. 4, pp. 29-32.

2. Vaitsekhovich S.M., Vlasov Iu.V. Razrabotka kompleksnoi sistemy otsenki effektivnosti proizvodstva i potrebleniia produktsii mashinostroeniia [Development of the complex evaluation system of production efficiency and consumption of engineering products]. PNRPU Sociology and Economics Bulletin, 2020, no. 2, pp. 146-147. 
3. Gusliakova G.P., Zhbannikov S.I. [et al.]. Povyshenie dolgovechnosti avtomobil'nykh metallicheskikh materialov [Improvement of the durability of automobile metal materials]. Nizhny Novgorod, 1991, 64 p.

4. Laskovyi A.A. Metodicheskie rekomendatsii po kompleksnoi otsenke effektivnosti mashinostroitel'nogo proizvodstva v NPO [Methodological recommendations for integrated assessment of the efficiency of machine-building production in a scientific-and-production association]. Kyiv, Naukova dumka, 1982, $121 \mathrm{p}$.

5. Vasil'ev G.A. Planirovanie povyshenii ekonomicheskoi effektivnosti mashinostroitel'nogo proizvodstva [Planning for increasing the economic efficiency of machine-building production]. Moscow, Mashinostroenie, 1985, $207 \mathrm{p}$.

6. Golosovskii S.I., Nikitin A.P., Tutov I.E. Tekhniko-ekonomicheskaia effektivnost' primeneniia perlitnykh stalei $\mathrm{v}$ energomashinostroenii [Feasibility of using perlitic steel in power plant engineering]. Ekonomicheskaia effektivnost' vnedreniia novykh tekhnologicheskikh protsessov $i$ konstruktsionnykh materialov $v$ tiazhelom mashinostroenii. Moscow, TsBTI, 1961, pp. 93-97.

7. Malenkovich V.B., Kuzhel' A.E. Novye metody otsenki effektivnosti tekhnologicheskikh protsessov $\mathrm{v}$ mashinostroenii [New methods for evaluating the effectiveness of technological processes in machine-building production]. Tekhnologiia, ekonomika, organizatsiia proizvodstva $i$ upravleniia: Ekspressinform. Moscow, TSNIIMASH, 1991, 18-1-91-03, pp. 1-7.

8. Metodicheskie polozheniia po vyboru parametrov, naibolee polno kharakterizuiushchikh potrebitel'skie kachestva produktsii i sviazannye s zatratami material'nykh i toplivo-energeticheskikh resursov [Guidelines for the selection of parameters that characterize consumer product quality and are associated with the cost of material and fuel-and-energy resources]. Moscow, Gosplan SSSR, NIIPiN, 1981, $32 \mathrm{p}$.

9. Erokhina L.S., Kalugina K.V., Mikhailov S.K. Metody prognozirovaniia razvitiia konstruktsionnykh materialov [Forecasting methods of the development of structural materials]. Leningrad, Mashinostroenie, 1980, $256 \mathrm{p}$.

10. Ivanov Iu.N., Tokarev V.V. Otsenivanie effektivnosti novykh tekhnologii po mezhotraslevym modeliam [Evaluating the effectiveness of new technologies by crossectoral models]. Modeli i metody v prognozirovanii nauchno-tekhnicheskogo progressa. Moscow, VNISI, 1984, no. 2, pp. 4-18.

11. Ivanov A.V., Ivanova V.A. Formirovanie i obnovlenie sistem izdelii [Developing and updating the product systems]. Moscow, Ekonomika, 1981, 56 p.

12. Kolesnichenko R.A. Progressivnye tekhnologii polucheniia zagotovok $i$ ekonomicheski tselesoobraznye granitsy ikh primeneniia [Advanced technologies for producing workpieces and economically feasible boundaries for their application]. Ekonomika mashinostroeniia. Kyiv, NAS of Ukraine, 1981, pp. 59-63.

13. Metodicheskie rekomendatsii po kompleksnoi otsenke effektivnosti meropriiatii, napravlennykh na uskorenie nauchno-tekhnicheskogo progressa [Me- 
thodological recommendations for the comprehensive assessment of the effectiveness of activities aimed at accelerating scientific-and-technological progress]. Moscow, USSR State committee for science and technology, 1988, no. 60/52, 120 p.

14. Pashko I.G., Pavlov V.I., Spektor A.N. Ekonomicheskii effekt novykh konstruktsionnykh materialov [The economic effect of new structural materials]. Moscow, Znanie, 1987, $64 \mathrm{p}$.

15. Poliak A.M. Povyshenie kachestva i uluchshenie ispol'zovaniia metallov [Improving the quality and application of metals]. Moscow, National University of Science and Technology (MISIS), 1980, $51 \mathrm{p}$.

16. Sadovnichaia S.G. Sozdanie normativov proizvodstvennykh zatrat na novuiu produktsiiu [Establishing standards for production costs of new products]. Ekonomicheskie problemy sokrashcheniia tsikla protsessa "issledovanierazrabotka-proizvodstvo". Proceedings of Sci.-Pract. Conf. Dnepropetrovsk, 1987, pp. $178-179$.

17. Savos'kin M.E. Snizhenie metalloemkosti izdelii putem primeneniia novykh materialov i tekhnologii [Reducing the metal consumption of products by applying new materials and technologies]. Kyiv, Naukova dumka, 1980, $11 \mathrm{p}$.

18. Malenkovnch V.B., Kuzhel' A.E. Issledovanie vzaimosviazi ekspluatatsionnykh parametrov novykh konstruktsionnykh materialov $\mathrm{s}$ ekonomicheskimi pokazateliami [Study of the relationship of the operational parameters of new structural materials with economic indicators]. Sinergetika. Novye tekhnologii polucheniia i svoistva metallicheskikh materialov. Proceedings of the 2nd All-Union Symposium, Moscow, Baikov Institute of Metallurgy and Materials Science, RAS, 1991, $176 \mathrm{p}$.

19. Filiptsev E.Ia., Danilin V.I. Model' opredeleniia srokov zameny izdelii novymi pri razrabotke piatiletnego plana ob"edineniia [A model for determining the terms for items replacement when developing a five-year plan]. Ekonomikomatematicheskie modeli v sisteme upravleniia predpriiatiem. Ed. N.P. Fedorenko, I.P. Shubkina. Moscow, Mashinosiroenie, 1983, pp. 148-160.

20. Terekhin V.I. Ekonomicheskoe obosnovanie obnovleniia produktsii [Business case for product updates]. Ekonomicheskie problemy mezhotraslevogo $i$ regional'nogo upravleniia nauchno-tekhnicheskim progressom. Leningrad, Palmiro Togliatti Leningrad Engineering and Economics Institute, 1986, pp. 90-99.

Оригинальность $99 \%$

Получено 10.12.2019 Принято 10.01.2020 Опубликовано 29.06.2020 


\section{S.M. Vaytsekhovich, Yu.V. Vlasov}

\section{A MODEL OF ECONOMIC EFFICIENCY OF STRUCTURAL MATERIALS PRODUCTION AND CONSUMPTION IN MACHINE BUILDING INDUSTRY}

The authors suggest a general economic criterion which links the process and operating parameters with economic indicators, representing costs feasibility for production and operation of technical system in temporal parameters of its lifetime. Presented is a model for economic analysis and evaluation of structural materials application. Assessment criterion for evaluating the economic efficiency of technological processes of the manufacturer includes the automation coefficient, dispersion, variation coefficient, which allows to identify the effective technology; those of the consumer - parts ratio, registration of repair and broken parts replacement. The assessment criterion allows one to reveal the most rational technology among the candidates, from the standpoint of level stabilization for physical and mechanical properties of materials under technological conversion. Based on the experimental data of new constructional materials development in power engineering, the tables of $K_{\sigma}$ conformity factor were generated by mathematic simulation, taking into account dependence of expenses on technological properties of component part.

The general economic criterion $\frac{K_{t} \cdot K_{N}}{K_{\sigma}}=$ const, cal and temporal dependences of separate parts included in the technical system concerned during the economic analysis thereof. The authors put forward the assessment model of economic expediency for manufacturing and operation of technical system by master unit, providing calculation of the minimum expenses considering parts work ratio, repair performed and part replacement in the course of technical system operation. A database of conformity coefficients $K_{T}$ has been developed, which takes into account the influence of technological properties level on the workpiece durability. A method is devised for determining the expected costs by upper and lower limits, allowing for the parts work ratio, repair costs and their complete replacement. The strategy is based on the comparison of the assessment criteria reflecting technical and economic indicators of alternative technologies and materials by revealing the rational costs of individual parts manufacturing and operation, taking into account additional costs of their replacement and repair ratio.

Keywords: obsolescence and physical deterioration, process cycle, conformity factor, economic expenditures and efficiency, master unit, inversed mode, ratio of component lifetime, technical system, downtime, machine stop of subsystem, optimization model, costs per unit, automation.

Sergey M. Vaytsekhovich - Candidate of Technical Sciences, Senior Researcher, Chief Researcher, Technomash Scientific and Production Association, e-mail: ask-mlad@mail.ru.

Yury V. Vlasov - Candidate of Technical Sciences, General Director, Technomash Scientific and Production Association, e-mail: info@tmnpo.ru.

Received 10.12.2019 Accepted 10.01.2020 Published 29.06.2020 